\title{
Téoros
}

Revue de recherche en tourisme

\section{Espaces protégés au Québec - Une ressource menacée}

\section{Nathalie Zinger}

Volume 14, numéro 1, printemps 1995

Les parcs : des réseaux en mutation

URI : https://id.erudit.org/iderudit/1077053ar

DOI : https://doi.org/10.7202/1077053ar

Aller au sommaire du numéro

Éditeur(s)

Université du Québec à Montréal

ISSN

0712-8657 (imprimé)

1923-2705 (numérique)

Découvrir la revue

Citer cet article

Zinger, N. (1995). Espaces protégés au Québec - Une ressource menacée.

Téoros, 14(1), 28-30. https://doi.org/10.7202/1077053ar d'utilisation que vous pouvez consulter en ligne.

https://apropos.erudit.org/fr/usagers/politique-dutilisation/ 


\title{
Espaces protégés au Québec - Une ressource menacée
}

\author{
Nathalie Zinger*
}

\section{La conservation de la biodiversité et l'engagement du Québec}

Ennovembre 1992, leQuébeca adhérépar décret du Conseil des ministres aux principes de la Convention sur la diversité biologique et élabore depuis une stratégie de mise en oeuvre. Une des orientations de cette stratégie est wd'établir et maintenir les aires protégées nécessaires à la conservation de la biodiversités entre autre en complétant les réseaux de parcs et de réserves écologiques et en favorisant la conservation des milieux naturels se trouvant dans des propriétés privées (Gaudreau, 1994).

Cet engagement s'inscrit dans un contexte planétaire qui recherche la construction d'une société durable visant à améliorer la qualité de vie des êtres humains tout en préservant la vitalité et la diversité de la Terre. Pour ce faire, plusieurs volets complémentaires devront garantir une utilisation durable des ressources renouvelables, assurer une gestion intégrée du territoire, préserver les systèmes entretenant la vie, restaurer et maintenir l'intégrité des écosystèmes et conserver la biodiversité (UICN, PNUE et WWF, 1991).

Il est donc essentiel de reconnaître la con= tribution d'un réseau complet d'aires protégées dans la représentation de la diversité biologique et de comprendre comment ces aires s'insèrent dans une stratégie de conservation s'appliquant à l'ensemble du territoire intégrant protection, utilisation multiple et gestion intégrée(Nelson, 1993; Noss, 1992; Theberge, 1993).

Tout en préservant des espèces rares et menacées, des communautés naturelles exceptionnelles, des habitats critiques, des écosystèmes complets et des paysages représentatifs, les sites qui excluent toute exploitation forestière, minière et énergétique contribuent à conserver notre «capital

- Madame Nathalle Zinger est directrice-Quebec, pour le Fonds mondial pour la nature Canada (WNF). natures et servent de zones de référence pour évaluer les impacts d'autres formes de développement.

\section{Les sites protégés au Québec}

La crétion des premiers parcs au Québec, soit les parcs nationaux des Laurentides et de la Montagne Tremblante, remonte à 1895. Il a fallu cependant attendre $1974 \mathrm{et}$ 1977 pour que soient adoptées la Lai sur les réserves écologigues et la Loi sur les pars. Dès 1986, un moratoire sur la création de parc a été instauré par l'ancien ministère du Loisir, de la Chasse et de la Pếche et il faudra attendre cinq ans avant qu'il ne soit levé et que la planification du réseau soit réactivée. Ainsi en 1992, un plan d'action quinquennal intitulé aLa nature en héritage» a été déposé et proposait la création de quatre parcs au suddu Québec et la mise en réserve de 18 territoires pour fin de parc au nord, quatre d'entre eux devant être constitués d'ici 1997 (MLCP, 1992). Ce redressement était fort attendu mais reste toutefois insuffisant pour assurer la représentation de la diversité biologique du Québec (Bourdages et Zinger, 1994).

Le tablenu 1 présente le réseau québécois d'espaces naturels protégés qui comprend principalement trois parcs nationaux, 17 parcs québécois et 49 réserves écologiques. Il existe plusieurs autres types de sites dont le degré de protection reste incomplet car l'exploitation commerciale de ressources forestières ou minières reste permise, ce qui est notamment le cas pour les vingt réserves fauniques, encore communément appelées «parcs», Il estroutefoisimportant de noter que depuis la levée du moratoire, vingt-cinq réserves écologiques et un parc ont été créés.

La contribution de ces sites protégés à la représentation de la diversité biologique a été évaluée par le Fonds mondial pour la nature dans le cadre de sa compagne pancanadienne «Espaces en dangerw. L'analyse a été fondée sur le découpage des 43 régions naturelles utilisé par le ministère del'Environnement et de la Faune dans sa planification du réseau de parcs et sur une méthodologie développée en collaboration avec le Conseil canadien des aires écologiques (WWF, 1993; WWF, in press). Comme l'indique le tablean 2, cinq régions naturelles sur 43 sont représentées ou modérément représentées tandis que neuf le sont partiellement et 29 peu ou pas du tout. Ceci nous indique qu'un important travail resteà accomplir pour atteindre une représentation adéquatedu patrimoine naturel québécois.

En somme, le portrait global de la préservation des milieux naturels du Québec demeure peu enviable, particulièrement lorsque celui-ci est comparé avec la situation dans les provinces canadiennes les plus populeuses. Ainsi, la proportion du territoire protégé au Québec est de 4,2\%, loin derrièrel'Aiberta $(9,5 \%)$, la ColombieBritannique $(7,8 \%)$ et l'Ontario $(5,5 \%)$ (WWF, 1993): Al'échelle canadienne, 4,8 $\%$ des terres et des eaux sont protégées, ce quiconfere au Canada unevingt-deuxième position à travers le monde (Theberge, 1993).

\section{Les défis}

Malgré les efforts déployés au cours des dernières années, quatre mythes entourant les milieux naturels au Québec persistent au détriment de la protection de la diversité biologique.

- Le Québec possède de vastes et de nombreux espaces protégés. Il suffit d'ouvrir une carteroutière du Québec pour découvrir d'immenses taches vertes qui sontinjustement associéesà des sites protégés, comme c'est le cas pour les réserves fauniques. En fait, le réseau québéoois est petit et éclaté et ne contribue pas à sa juste valeur à la protection de la diversité biologique du Québec.

- Les sites protégés sont considérés comme un luxe dans une économieen détresse. Pourtant, une économie saine et un environnement en santé vont de pair et sont indissociables; l'état des pêcheries dans l'Est du Québec en étant un exemple flagrant. 
Ainsi, les sites protégés et leur valeur «nature» peuvent contribuer significativement à la diversification de l'économie québécoise. Il est nécessaire toutefois de comprendre les bénéfices que nous procure la nature et quels sont les coûts associés aux décisions que nous prenons face à l'allocation des ressources, que ce soit pour leur exploitation industrielle ou pour leur protection (WWF, 1993). L'augmentation au cours des dernières années du nombre de personnes impliquées dans des activités reliées à la faune et le développement du tou= risme de nature ne sont que deux indicateurs du rôle économique que peuvent jouer nos sites protégés.

- Iln'y a aucune urgence pour protéger notre patrimoine naturel, et pourtant au Canada, $1 \mathrm{~km}^{2}$ de nature sauvage disparait toutes les heures, $85 \%$ des terres humides ont été drainées, $87 \%$ des prairies sauvages ont été converties à l'agriculture et $65 \%$ des forêts productives sont destinées à l'exploitation. Le Canada et le Québec font partie des raresjuridictions à travers le monde où le parachèvement d'un réseau représentatif de la diversité biologique est encore possible, mais plus le temps passe, plus il sera difficile de le faire, surtout dans les régions les plus peuplées comme au sud du Québec.

- Les Québécois ne s'intéressent pas à l'avenir de leur patrimoine naturel. Pourtant, un sondage mené en mars 1994 par Environics a démontré que $78 \%$ des Québécois appuient la protection du milieu naturel et sont d'avis que le gouvernement du Québec se doit de tenir l'engagement, pris en novembre 1992 , de parachever, d'ici l'an 2000, un réseau de sites protégés représentatifdela diversité biologique du Québec. Cet appui était d'ailleurs le plus massif à travers le Canada. De plus, 40000 Québécois ont signé la Charte canadienne des sites naturels développéepar le Fonds mondial pour la nature dans le cadre de la campagne «Espaces en dangers.

Par delà ces mythes, le défi ultime pour le Québec est celui d'atteindre le but qu'il s'est fixé et d'adopter des stratégies et des échéanciers lui permettant de parachever un réseau intégré d'aires protégées d'ici l'an 2000. Il est donc impératif d'analyser les carences du réseau actuel de sites pro-

\section{TABLFAU 1}

\section{ESPACES NATURELS PROTÉGÉS AU QUÉBEC}

\begin{tabular}{|c|c|c|}
\hline \multirow{2}{*}{\multicolumn{2}{|c|}{ * Parcs de conservation (11) }} & \multirow{13}{*}{$2500 \mathrm{~km}^{2}$} \\
\hline & & \\
\hline Aiguebelle & $241,7\left(\mathrm{~km}^{2}\right)$ & \\
\hline Bic & 33,2 & \\
\hline Gaspésie & 801,7 & \\
\hline Grands-Jardins & 310,0 & \\
\hline Île-Bonaventure et Rocher-Percé & 5,8 & \\
\hline Jacques-Cartier & 670,6 & \\
\hline Miguasha & 0,6 & \\
\hline Mont-Megantic & $54,7(*)$ & \\
\hline Mont-Saint-Bruno & 5,9 & \\
\hline Pointe-Taillon & 92,2 & \\
\hline Saguenay & 283,6 & \\
\hline \multicolumn{3}{|l|}{ * Parcs de récréation $(\omega)$} \\
\hline Frontenac & $155,3\left(\mathrm{~km}^{2}\right)$ & \\
\hline Îles-de-Boucherville & 8,2 & \\
\hline $\begin{array}{l}\text { Mont-Orford } \\
\text { Mont-Tremblant }\end{array}$ & $\begin{array}{r}38,4 \\
1490,0\end{array}$ & \\
\hline Oka & 23,7 & \\
\hline Yamaska & 12,9 & \\
\hline \multirow{8}{*}{$\begin{array}{l}\text { * Parcs nationaux ( } 3 \text { ) } \\
\text { Archipel de Mingan } \\
\text { Forillon } \\
\text { Mauricie } \\
\text { * Réserves écologiques (49) } \\
\text { * Autres sites - liste non exhaustive } \\
\text { Sites sur terres prives (43) } \\
\text { P. Gatineau et autres sites (7) } \\
\text { Futures réserves écologiques (23) }\end{array}$} & & $934 \mathrm{~km}^{2}$ \\
\hline & $150,0\left(\mathrm{~km}^{2}\right)$ & \\
\hline & 240,4 & \\
\hline & $543,9(*)$ & \\
\hline & (") & $1664 \mathrm{~km}^{2}$ \\
\hline & $136,0\left(\mathrm{~km}^{2}\right)$ & \\
\hline & 376,0 & \\
\hline & $1151,8\left({ }^{\mathrm{Nm}}\right)$ & \\
\hline
\end{tabular}

\section{SITES AVEC UN STATUT OFFRANT UNE PROTECTION INCOMPLÈTE}

\begin{tabular}{lr}
\hline * Reserves fauniques (20) & $68513 \mathrm{~km}^{2}$ \\
* Projets de parc au nord du 50e parallèle (18) & $57720 \mathrm{~km}^{2}$ \\
* Projet du parc marin du Saguenay (parc provincial/fédéral) & $1138 \mathrm{~km}^{2}$ \\
* Habitats fauniques (490) & $27600 \mathrm{~km}^{2}$ \\
* Réserves nationales de faune (8) & $660 \mathrm{~km}^{2}$ \\
Refuges d'oiseaux migrateurs (33) & \\
\hline
\end{tabular}

Source: WWF (1993); (") mises à jour, janvier 1995.

tégés quant à la représentation de la biodiversité, d'identifier les sites candidats potentiels pouvant combler ces lacunes et d'élaborer un plan de réseau intégré d'ici la fin de 1995; des objectifs réalisables, les données, l'expertise et la méthodologie étant disponibles. L'élaboration d'un cheminement critique identifiant des objectifs de réalisation tel le nombre de régions naturelles à représenter annuellement permettra d'évaluer le progrès accompli sur le terrain tout au long des cinq prochaines années. Desmesuresincitatives pour protéger les milieux naturels sur des terres privées, des mécanismes de protection intérimaire et des alternatives de financement seront également nécessaires pour assurer une intégration des aires protégées, privées et publiques, au sein d'un réseau représentauf.

Le territoire québécois étant immense, des stratégies diffêrentes seront nécessaires pour représenter la diversité biologique du sud et du nord du Québec. Pour le sud, où les pressions de développement sont les plus fortes et où la majorité des Québécois demeurent, la contribution des terres privées et des territoires municipalisés sera vitale. Une participation accrue d'organismes non gouvernementaux et de particuliers par le biais de fiducies foncières et de réserves privées, une confirmation réglementée de la vocation de conservation et de protection des parcs régionaux et une clarification quant à leur contribution à la représentation de la biodiversité et une consolidation du statut de protection des réserves fauniques, tel que projeté à Plaisance en Outaouais, sont des moyens réalistes pour parachever le réseau de sites protégés au sud. Par ailleurs, il est essentiel également d'assurer l'intégrité écologique des sites déjà existant ou projetés en assurant des modes de gestion conformes aux 
TABLEAU 2 -
REPRÉSENTATION DES REGIONS NATURELLES AU QUÉBEC

Régions naturelles représentées (2)

\# Région naturelle

B20 Fjord du Saguenay

L 9 Collines montérégiennes

Site(s) protégé(s)

P. conservation Saguenay

P. conservation St-Bruno

(Mont-St-Hilaire)

Régions naturelles modérément représentées (3)
\# Région naturelle
A3 Massif gaspésien
B22 Massif laurentides Nord
L13 Cuestas de la Côte Nord
Site(s) protégé(s)
P. conservation Gaspésie
P. national Forillon
P. conservation Grands-Jardins
P. conservation Jacques-Cartier
P. national Archipel Mingan

Régions naturelles partiellement représentées (9)

$\begin{array}{lll}\# & \text { Région naturelle } & \text { Site(s) protégé(s) } \\ \text { A1 } & \text { Iles de la Madeleine } & \text { (Réserve écologique île Brion } \\ \text { A5 } & \text { Estrie, Beauce, Bellechasse } & \text { P. récrétion Frontenac } \\ \text { A7 } & \text { Monts Sutton } & \text { P. récrétion Mt-Orford } \\ \text { B17 } & \text { Laurentides boréales } & \text { P. récréation Mt-Tremblant } \\ \text { B19 } & \text { Basses-terres Sague. / L.St-Jean } & \text { P. conservation Pte-Taillon } \\ \text { B23 } & \text { Laurentides méridionales } & \text { P. national Mauricie } \\ \text { B26 } & \text { Ceinture argileuse Abitibi } & \text { P. conservation Aiguebelle } \\ \text { L10 } & \text { Basses-terres St-Laurent } & \text { P. récréation Boucherville } \\ & & \text { P. récrétion Oka } \\ \text { L11 } & \text { Littoral sud de l'estuaire } & \text { projet de parc - Plaisance }\end{array}$

Régions naturelles non ou peu représentées (29)

$\begin{array}{lll}\# & \text { Région naturelle } & \text { Site (s) protégé(s) } \\ \text { A2 } & \text { Baie des chaleurs } & \text { P. conservation Bonaventure }\end{array}$

A4 Monts Notre-Dame

A6 Montagnes frontalières

B18 Massif du mont Valin

B21 Côte de Charlevoix

B24 Vallée de la Gatineau

B25 Basses-terres Témiscamingue

L8 Basses-terres appalachiennes

L12 Plaine $\mathrm{H}_{+}+\mathrm{M}$. Côte-Nord

L14 Ile d'Anticosti

B16, $\quad 19$ régions naturelles au nord

$\mathrm{B} 27$ a du 50 e parallèle

projet de parc - Mt-Mégantic (1)

projet de parc - Mt-Valin

(P. de la Gatineau)

P. récréation Yamaska

projet de parc - Vauréal

18 projets de parc pouvant représenter 16/17 régions naturelles

343

L15

(1) Le parc du Mont-Mégantic a été créé en 1994. L'évaluation de sa contribution à la représentation de la région naturelle (AG) reste à compléter.

N.B. Bien que non présentées dans le tableau, les données sur les réserves écologiques et sur certains sites privés ont été incorporées dans l'analyse faite pour identifier le niveau de représentation des régions naturelles.

Sources: Bourdages et Zinger (1994); WWF (1993).

vocations de conservation et d'éducation des sites.

Dix-huit territoires ont été mis en réserve pour fin de parc au nord du cinquantième parallèle. L'inclusion de ces projets au plan d'affectation des terres publiques et l'intégration des préoccupations et des intérêts des Premières Nations sont essentielles. Seul un partenariat étroit établi dès le début du processus de planification per- mettra de protéger un échantillon représentatif de la diversité biologique du nord québécois.

Ayant un des littoraux les plus longs au Canada, le Québec devra éventuellement se pencher sur la oonservation d'aires marines. Le projet du parc marin SaguenaySaint-Laurent est un point de départ important bien que sa création légale tarde toujours malgré les consultations publi- ques sur les limites du parc et sur son plan directeur dont le dépôt est imminent.

\section{Conclusion}

Dans ses réponses à un questionnaire préélectoral sur un plan d'action pour les parcs et les sites protégés au Québec, le Parti Québécois a identifié la mise sur pied d'un véritable réseau de protection des espaces naturels comme étant la clé de la mise en oeuvre de la Convention sur la biodiversité ets'estengagéà ce quece réseau représente adéquatement la diversité des régions naturelles québécoises.

Une meilleure compréhension du rôle des sites protégés dans le processus d'affectation des terres et d'allocation des ressources renouvelables, la dissipation des mythes entourant la protection des milieux naturels, l'établissement d'un plan de réseau et d'un cheminement critique de réalisation et un appui solide des Québécois sont essentiels pour assurer la conservation de la diversité biologique du Québec.

Cet ambitieux projet de société demeure réalisable. Il ne faut surtout pas manquer une chance unique et quoi de mieux que de célébrer en 1995 le centenaire des pares québécois tout en jetant les fondations pour les prochains cent ans. $f$

\section{RÉFÉRENCES}

BOURDAGES, J.-L. et N. Zinger, La conservation dest paysages naturels au Qudbec: mythe ou realite? Trames, no 9, 1994, pp. 66-71.

GAUDREAU, Léopold, Convention sur la diversité biologique - Stratégle de mise en oeuvre au Québec (état de la situation), allocution présentée le 4 novembre 1994 a l'Association des biologistes du Québec, Quebec, 1994, 16 p.

MLCP. La nature en heritage - Plan d'action sur les parcs, Gouvernement du Québec, Québec, 1992. $22 \mathrm{pl}$

NELSON, J.G., Beyond Panks and Protected Areas, in DEARDEN, P. \& R. ROLLINS (Ed.), Parks and Protected Areas In Canada, Toronto, Oxilord University press, 1993, pp. 45-56.

NOSS, Feed F. The Wildands Project - Land Conserwation Strategr, Wild Earth, Special issue on The Wilcdlands Project, 1992, pp. 10-25.

THEBEAGE, J.B., Ecology, Conservation, and Protected Areas in Canada, in DEARDEN, P. \& R. ROLLINS (Ed.). Parks and Protected Areas in Canada, Toronto. Oxford University press, 1993, pp. 137. 153.

UICN, PNUE, WWF, Sauver la Planete - Stratbgio pour Iavenir de la vie, Gland, Suisse, 1991, 250 p.

UQCN, Les parcs du Québec, numéro spécial de FraneVert, vol. 10, no 3, 1993, 38 p.

WWF, Rapport d'étape $n^{\circ} 4$, rapport annuel de la campagne *Espaces en danger* produit par le Fonds mondial pour la nature Canada, Toronto, 1993, 48

WWF. A Protected Areas Gap Analysis Methodology. rapport scientifique produit dans le cadre de la campagne a Espaces en dangers. Toronto lin press). 